\title{
CAPACIDAD PARA BIOACUMULACIÓN DE COBRE Y ZINC DE ESPECIES SILVESTRES QUE SE DESARROLLAN SOBRE UN RELAVE MINERO EN CORCONA - HUAROCHIRÍ
}

\author{
CAPACITY FOR BIOACCUMULATION OF COPPER AND ZINC OF WILD SPECIES THAT DEVELOP ON A MINING \\ RELAVE IN CORCONA - HUAROCHIRÍ
}

https://doi.org/10.52109/cyp202119

Jem Valenzuela', José Eloy Cuellar²

${ }^{1}$ Ing. Ambiental, Universidad César Vallejo.

2 Doctor, Profesor Principal de la Universidad Nacional Agraria La Molina.

\section{REGISTROS}

Recibido el 15/08/2020

Aceptado el 30/09/2020

Publicado el 31/01/2021

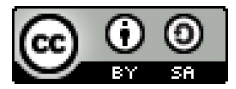

\section{PALABRAS CLAVE}

Relave Minero, Bioacumulación, Cobre,

Zinc.

\section{KEYWORDS}

Mining waste, Bioaccumulation, Copper,

Zinc.

\section{RESUMEN}

La presente investigación tuvo como objetivo general, determinar si las plantas que crecen de manera silvestre sobre el relave minero de Corcona, Huarochirí, Lima, tienen capacidad de bio-acumular cobre y zinc, para lo cual se evaluaron las especies silvestres que están creciendo sobre dicho relave, estas especies fueron recogidas a través de un muestreo estratificado en el cual se tuvieron criterios de inclusión como la presencia de especies y la alta densidad vegetativa y de exclusión fue la alta pendiente, luego de seleccionado el cuadrante a trabajar se realizó un muestreo al azar y se analizó en el laboratorio mediante el método de espectrofotometría de absorción atómica el cual es el método más adecuado para análisis de micro elementos y de elementos traza como el cobre y el zinc, obteniendo como resultado que la especie Crotón alnifolius tiene una alta capacidad de bio-acumulación de zinc llegando a almacenar en sus estructuras $2338.52 \mathrm{ppm}$ por lo que esta especie puede ser utilizada como especie fitorremediadora de suelos en otros lugares con suelos contaminados por zinc, procurando que dicho lugar tenga características similares a las del relave estudiado.

\section{ABSTRACT}

The present investigation was to determine if overall objective plants growing on mine tailings Corcona Huarochirí, Lima, have high capacity Bio-accumulate copper and zinc, for which the population will have the mining waste that left the company Peru Bar and whose shows are those species that grow on this tailings, these species were collected through a show laminate in which inclusion criteria were the presence of species and high density vegetative and exclusion was the high slope, then selected the quadrant to work a random sampling was performed and analyzed in the laboratory by the method of atomic absorption spectrophotometry which is the most suitable method for analysis of microelements and trace elements such as copper and zinc. the results showsthat the specie Croton alnifolius has high capacity for bio-accumulation of zinc reaching store in their $2338.52 \mathrm{ppm}$ structures so that this species can be used as fitorremediadora kind of soil in other sites contaminated by zinc, making sure that the place look similar to the tailings studied soil characteristics. 


\section{INTRODUCCIÓN}

La minería es la actividad económica que mayores dividendos producen a nuestro país. Según la Sociedad Nacional de Minería, Petróleo y Energía, en su publicación Impacto Económico de la Minería en el Perú del año 2012 afirma que esta actividad es considerada el principal sector exportador del País, debido a que representa el $59 \%$ de las exportaciones totales. Sin embargo, no solo genera beneficios a las poblaciones más cercanas a las zonas en donde se desarrolla esta actividad, en la gran mayoría de los casos, también ha traído problemas de diversas índoles. Como sabemos la minería trae consigo muchos problemas ambientales, ya sea la contaminación de ríos, lagos, lagunas o cuerpos de agua, muchos de los cuales hoy ya no existen. También podemos mencionar la contaminación del aire, sin dejar de lado la del suelo que ha provocado la degradación de áreas agrícolas y ganaderas. Una de las características de la contaminación de suelos por minería es el alto contenido de metales pesados, los cuales se encuentran por encima de los parámetros establecidos. Este daño directo sobre el medio ambiente perjudica indirectamente al hombre, generando los problemas siguientes: falta de alimentos, patologías respiratorias y oftalmológicas entre la población circundante, además de problemas nerviosos como stress generados por ruidos, vibraciones, polvo y cambios del paisaje" (Brack \& Mendiola, 2004).

La presente Investigación tiene por finalidad analizar las especies nativas que se desarrollan sobre el relave minero ubicado en la provincia de Huarochirí, región Lima, dejo hace más de 10 años; relave que se encuentra, según el ministerio de energía y minas, entre los 5551 relaves mineros sin tratamiento en todo el territorio peruano, para lo cual se realizaron diversas evaluaciones de campo: un análisis de biomasa para identificar cuáles son las cinco especies con mejor respuesta de adaptación al medio, luego se hicieron análisis para determinar el contenido de cobre y zinc mediante un análisis por espectrofotometría de absorción atómica que nos indicará el contenido de dichos metales en partes por millón (ppm) para luego seleccionar la especie con mayor cantidad de cobre y zinc. Se realizarán 3 repeticiones y una muestra control, cuyos datos determinarán si esta especie podría considerarse bioacumuladora.

Para lo cual se formuló los siguientes problemas como problema general se tiene: ¿Cuál es la capacidad para bio-acumular cobre y zinc de las especies vegetales que crecen en el relave minero Corcona Huarochirí? De los cuales se desligan los siguientes problemas específicos: ¿Cuáles son las especies capaces de desarrollarse sobre el pasivo minero en Corcona?, Entre las especies que crecen sobre el relave minero ¿Cuáles son las que mayor adaptación al medio tienen?, ¿Cuál es la especie con mayor concentración de cobre y zinc? Y ¿Pueden estas plantas servir como especies fitorremediadoras de suelos en otros relaves?

Como hipótesis se afirma que Las especies vegetales que se desarrollan sobre el relave minero en Corcona, tienen alta capacidad para bio-acumular cobre y zinc. 
Se tiene como objetivo general: determinar si las plantas que crecen sobre el relave minero de Corcona tienen alta capacidad de Bio-acumular cobre y zinc. Además, como objetivos específicos la intención es: identificar las especies que se desarrollan sobre el relave minero en Corcona; identificar la especie o las especies que mayor adaptación al medio presentan; identificar la especie con mayor concentración de Cobre y zinc; y proponer una especie natural para su uso como planta fitorremediadora de suelos contaminados por relaves mineros.

\section{MATERIALES Y MÉTODOS}

\section{Ubicación del área de estudio}

El distrito de Santa Cruz de Cocachacra, en sus inicios pertenecía al Distrito de San Bartolomé. Habitado desde 1978, al principio su población era pequeña. Sin embargo, gracias al crecimiento de su población y al desarrollo de sus actividades industriales llegó a alcanzar, con el tiempo, la categoría de distrito. Pese a ser rica en producciones agrícolas, como en el cultivo de manzanas, maíz y algodón, la actividad de mayor importancia en el pasado fue la minería; es por ello que existen vestigios de dicha actividad a lo largo del pueblo de Corcona.

\section{Figura 1}

\section{Ubicación de "Corcona" en el distrito de Santacruz de Cocachacra, Huarochiri, Lima}

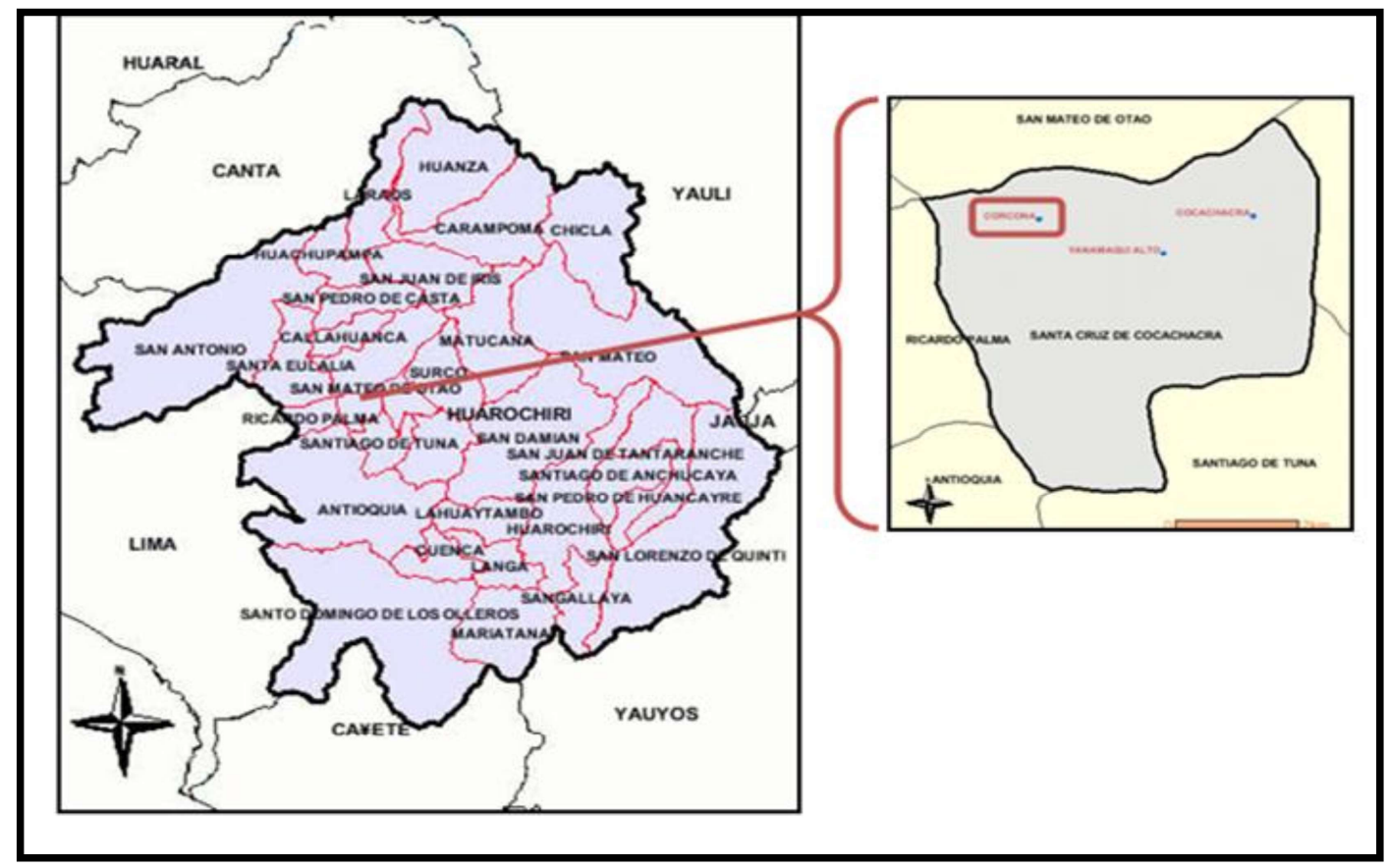

\section{Ubicación del pasivo minero}

Se encuentra ubicado a 1250 metros sobre el nivel del mar, en el Km. 49.5 de la carretera central, en el paraje de Corcona, distrito de Santa Cruz de Cocachacra, provincia de Huarochirí, en la región Lima. En este lugar la empresa minera operó 
desde el año 1960 hasta el año 2001, explotando en un inicio baritina y luego, a partir del año 1975, zinc, cobre y oro.

\section{Metodología}

Las técnicas e instrumentos utilizados en la presente investigación fueron:

Técnicas

- Observación de Campo

- Muestreo de suelos

- Muestreo de especies

Instrumentos

- Fichas de recolección de datos.

- Autocad, Google Earth.

- Materiales y equipos de campo.

\section{Selección de los puntos de muestreo}

Luego de delimitar el área total del relave, se determinó que el relave tenía un área total de 5'212,044.073 m2 y un perímetro igual a 11,808.60 m. Luego se procedió a realizar transectos de $500 \times 500 \mathrm{~m}$.

De los 31 cuadrantes obtenidos, se seleccionó uno en base a: la pendiente, accesibilidad de la zona y presencia de especies vegetales. Se seleccionó el cuadrante número 19.

Luego se definieron cuatro intervenciones las cuales estuvieron divididas en cuatro visitas: las dos primeras para el conteo de individuos por especie, donde se procedió a la toma de muestras de suelo para determinar el contenido de humedad, y la de muestras botánicas de las 5 especies que mayor dominancia presentaba con la finalidad de la identificación por nombre científico y familia. Las dos visitas siguientes se llevaron a cabo para realizar la extracción de las especies y suelo con la finalidad de realizar los análisis en laboratorio para determinar el contenido de cobre y zinc que almacenan en sus estructuras.

\section{Muestreo para las especies y suelo}

De acuerdo con el manual de métodos básicos de muestreo y análisis en ecología vegetal, se realizó el muestreo "aleatorio estratificado" que consiste en separar en subgrupos o estratos que tienen cierta homogeneidad. Después de la separación, dentro de cada subgrupo se realizó un muestreo aleatorio simple para escoger sitios para el muestreo.

\section{Muestreo del sitio control (muestra de suelo y plantas)}

Los sitios de control son importantes para entender el valor de la información de muestreo. Se seleccionaron lugares con características comunes, no contaminadas, cerca al lugar de muestreo del sitio contaminado. 
Los sitios de control son importantes para entender el valor de la información de muestreo. Se seleccionaron lugares con características comunes, no contaminadas, cerca al lugar de muestreo del sitio contaminado.

\section{Fase de campo, primera visita}

Tuvo como finalidad determinar la especie que tenía mejor adaptación al medio y observar el comportamiento de dichas especies conforme al tiempo:

- Se delimitó el área a estudiar conforme a las coordenadas obtenidas desde Google Earth. Se procedió a seleccionar 4 cuadrantes de $1 \times 1 \mathrm{~m}$ en áreas con mayor vegetación, en dichos cuadrantes se procedió a contar el número total de individuos por especie.

- Se extrajeron las 5 especies más abundantes en los 4 cuadrantes y se extrajo una muestra de suelo por cada especie del mismo lugar de donde se extrajo la especie.

\section{Fase de campo, segunda visita}

En esta nueva intervención se buscaba observar el estado en el cual se encontraba las especies debido al cambio de clima:

- En los 4 cuadrantes seleccionados anteriormente se realizó un nuevo conteo, que consistió en enumerar las especies vivas.

\section{Fase de campo, tercera visita}

Esta vez se extrajeron las 5 muestras de especies y de suelo con la finalidad de realizar el análisis en laboratorio para determinar el contenido de cobre y zinc acumulado en la planta y una muestra control.

- Del cuadrante de 500 m2 obtenido en Google Earth se realizó el muestreo al azar de las 5 especies vegetales. Para ello se extrajo la especie desde la raíz procurando no maltratarla.

- Luego se recolectó la muestra de suelo de las especies. Se tomó una muestra de suelo en donde no había presencia de especies para determinar el contenido de cobre y zinc del relave. Para dicha muestra se excavaron 3 calicatas y se extrajo 300 gr. de suelo y se combinó para obtener una sola muestra uniforme del relave.

\section{Fase de campo, cuarta visita}

Luego de haber obtenido los resultados del análisis de las especies y suelo, e identificar la especie con mayor capacidad de acumular cobre y zinc en su estructura.

- En el cuadrante seleccionado, se realizó el muestreo al azar de individuos de la especie Croton Alnifolius, quien fue la que, según los análisis realizados en laboratorio, demostró tener mayor contenido de Cobre y zinc, lo cual se puede observar en el cuadro de resultados. 
- Se recolectó una muestra de suelo no contaminado la cual sirvió como muestra patrón. Luego de extraer las plantas desde su raíz se recolectó la muestra de suelo de las especies.

Fase de laboratorio, determinación de humedad

- En el laboratorio se procedió a moler las muestras de suelo y tamizarlas hasta un tamaño menor a 220 micras.

- Luego se pesó 35 gr. de cada muestra, luego llevar las 6 muestras a la estufa y secar a $85^{\circ} \mathrm{C}$ por 24 horas.

\section{Determinación de Contenido de Cobre y Zinc en Plantas y Suelo}

La determinación de cobre y zinc en suelos y plantas se realizó en el laboratorio de química orgánica de la facultad de ingeniería química de la universidad Nacional de Ingeniería (UNI). Para lo cual se siguiendo el protocolo de métodos de análisis para suelos y lodos de la Universidad de Concepción. Facultad de Agronomía, Chile.

\section{RESULTADOS}

\section{Identificación de las especies por nombre y familia}

Tabla 1

Nombre científico, común y familia de las especies

\begin{tabular}{clll}
\hline Especies & \multicolumn{1}{c}{ Nombre Científico } & \multicolumn{1}{c}{ familia } & \multicolumn{1}{c}{ Nombre Común } \\
\hline Especie 1 & Croton alnifolius & Euphorbaceae & Croton amarillo \\
Especie 2 & Atriplex rotundifolia & Chenopodiaceae & Cachisco \\
Especie 3 & Althernantera Halimifolia & Amaranthaceae & Hierba blanca \\
Especie 4 & Bacharis Latifolia & Asteraceae & Chilca \\
Especie 5 & Capparis sp & Caparaceae & NN \\
\hline
\end{tabular}

\section{Densidad de la Biomasa}

Tabla 2

Índice de abundancia en la primera visita - julio

\begin{tabular}{|c|c|c|c|c|c|}
\hline \multirow{2}{*}{ Especie } & \multicolumn{4}{|c|}{ Número de repeticiones de la especie } & \multirow{2}{*}{ Total } \\
\hline & Cuadrante 1 & Cuadrante 2 & Cuadrante 3 & Cuadrante 4 & \\
\hline Croton alnifolius & 2 & 3 & 1 & 1 & 7 \\
\hline Atriplex rotundifolia & 1 & 1 & 2 & - & 4 \\
\hline $\begin{array}{l}\text { Althernantera } \\
\text { Halimifolia }\end{array}$ & - & 1 & 2 & - & 3 \\
\hline Bacharis Latifolia & - & 1 & - & 1 & 2 \\
\hline Capparis sp. & - & - & 1 & 1 & 2 \\
\hline Total & 3 & 6 & 6 & 3 & 18 \\
\hline
\end{tabular}


Tabla 3

Índice de abundancia en la segunda visita - septiembre

\begin{tabular}{lcccccc}
\hline \multirow{2}{*}{ Especie } & \multicolumn{5}{c}{ Número de repeticiones de la especie } & \multirow{2}{*}{ Total } \\
\cline { 2 - 6 } & Cuadrante 1 & Cuadrante 2 & Cuadrante 3 & Cuadrante 4 & \\
\hline Croton alnifolius & 2 & 1 & 1 & 1 & 5 \\
Atriplex rotundifolia & 1 & - & 2 & - & 3 \\
Althernantera & - & 1 & 2 & - & 3 \\
Halimifolia & - & 1 & - & 1 & 2 \\
Bacharis Latifolia & - & - & 1 & - & 1 \\
Capparis sp. & - & 4 & 6 & 2 & 15 \\
Total & 3 & & & & & \\
\hline
\end{tabular}

\section{Humedad}

Los resultados de humedad obtenidos determinaron que la especie de mayor abundancia tenía mejor humedad en el suelo, lo que podría significar la mejor proliferación de esta.

Tabla 4

Contenido de humedad en muestras de suelo

\begin{tabular}{lc}
\hline \multicolumn{1}{c}{ Especie } & \% de humedad del suelo \\
\hline Croton alnifolius & $7.30 \%$ \\
Atriplex rotundifolia & $4.48 \%$ \\
Althernantera Halimifolia & $3.58 \%$ \\
Bacharis Latifolia & $2.28 \%$ \\
Capparis Sp. & $2.90 \%$ \\
Relave & $1.64 \%$ \\
\hline
\end{tabular}

\section{Contenido de cobre y zinc en plantas y suelo}

Tabla 5

Contenido de cobre y zinc en las 5 especies

\begin{tabular}{lcc}
\hline \multicolumn{1}{c}{ Suelo de especie } & Cobre $(\mathrm{ppm})$ & Zinc $(\mathrm{ppm})$ \\
\hline Croton alnifolius & 88.4 & 855.5 \\
Atriplex rotundifolia & 164.9 & 6431.7 \\
Althernantera Halimifolia & 140.1 & 3416.5 \\
Bacharis Latifolia & 1143.4 & 17042.2 \\
\hline Capparis Sp. & 1307.3 & 21954 \\
Relave & 1279.9 & 25075 \\
\hline
\end{tabular}


Tabla 6

Contenido de cobre y zinc en el suelo de las 5 especies

\begin{tabular}{lcc}
\hline \multicolumn{1}{c}{ Especie } & Cobre $(\mathrm{ppm})$ & Zinc $(\mathrm{ppm})$ \\
\hline Croton alnifolius & 88.4 & 855.5 \\
Atriplex rotundifolia & 164.9 & 6431.7 \\
Althernantera Halimifolia & 140.1 & 3416.5 \\
Bacharis Latifolia & 1143.4 & 17042.2 \\
Capparis Sp. & 1307.3 & 21954
\end{tabular}

\section{Determinar si la especie es acumuladora}

Tabla 7

Contenido de cobre y zinc en las repeticiones de Croton Alnifolius

\begin{tabular}{lcc}
\hline \multicolumn{1}{c}{ Especie } & Cobre $(\mathrm{ppm})$ & Zinc $(\mathrm{ppm})$ \\
\hline Repetición 1 & 70.0 & 1930.9 \\
Repetición 2 & 64.14 & 1746.26 \\
Repetición 3 & 66.40 & 2244.19 \\
Repetición 4 & 66.93 & 2338.52 \\
Promedio & 66.9 & 2065.0 \\
\hline
\end{tabular}

\section{DISCUSIÓN}

Como se puede apreciar, en la tabla 7 existen plantas que tienen capacidad de bioacumular cobre y zinc teniendo las siguientes cifras: para la especie Crotón alnifolius almacena 70.0 ppm para cobre y 1930.9 ppm para zinc, con lo cual se podría decir que la hipótesis ha sido comprobada positivamente de acuerdo con los datos obtenidos.

Entre las especies presentes con gran abundancia hemos podido observar las siguientes: Crotón alnifolius, Atriplex rotundifolia, Alternanthera halimifolia, Bacharis Latifolia y Capparis Sp. de acuerdo con Holdrige (1967), la zona de vida que le pertenece al área de estudio es Desierto desecado Premontano tropical (Dd-Pt), zona en la cual la presencia de estas especies es común, de acuerdo con la biotemperatura media anual que es igual a $17.4{ }^{\circ} \mathrm{C}$. Con una precipitación de 139.7 milímetros, son áreas áridas o desiertas con pocas especies que viven de la humedad atmosférica. Por ello, las especies se encontraban marchitas durante el mes de septiembre, mes en el cual, según el Servicio Nacional de Meteorología e Hidrología (SENAMHI), la humedad atmosférica es menor.

De acuerdo con Espinoza (2010), la concentración de zinc que debe tener una planta en su estructura debe ser igual a de 27 a 150 ppm en estado natural, si contiene de 10 a 20 ppm se considera que la planta tiene déficit de carbono, y de 100 a 400 ppm es excesivo o tóxico para la especie. Para el cobre los rangos se consideran en déficit 
aquellas que contengan de 2 a 5 ppm, de 5 a 30 ppm normal o suficiente, y de 20 a 100 tóxico. Sin embargo, Baker (2001) afirma que existen especies que son capaces de tolerar concentraciones altas de metales pesados, por lo que estas especies se considerarían indicadoras, acumuladoras o hiperacumuladoras de acuerdo con la concentración que almacenen. En el caso de Zn, Jafre (2010) considera que es una especie hiperacumuladora si en su estructura almacena una cantidad mayor a 3000 ppm., y se considera acumuladora si contiene de 1500 ppm a 3000 ppm. El promedio de las repeticiones obtenidas es igual a 2065 ppm, por lo que esta especie se considera acumuladora de zinc, sin embargo, el promedio obtenido para cobre fue de 66.9 ppm. De ello se concluye que la especie Croton alnifolius podría servir como especie fitorremediadora para suelos contaminados con cobre.

Se sabe por los tratados de fisiología vegetal, que la habilidad de tolerar la presencia de metales pesados está determinada por el nivel de variación genética del individuo. Se puede determinar si se trata de plantas metal exclusivas o acumulativas si se observan algunas características tales como: el crecimiento dentro y fuera del área contaminada (Durand, 2010), ya que se pudo observar que las plantas que crecieron dentro del relave tienen un tamaño mucho menor al de las plantas que crecieron fuera de él. Se afirma que la especie Crotón alnifolius cumple dicha característica.

De las 5 especies encontradas sobre el relave Croton alnifolius, Atriplex rotundifolia, Alternanthera halimifolia, Bacharis Latifolia y Capparis Sp. se puede definir que la especie que mejores resultados nos dio fue: el Croton alnifolius, la cual es una especie descubierta y descrita por Macbride en el año 195. Esta puede desarrollarse entre las altitudes de 0 a 1500 m.s.n.m. teniendo como rango de distribución Arequipa, Lima Cajamarca y La Libertad. Además, se le considera una especie endémica (Chicago Field Museum, 2013), con lo cual podemos mencionar que la investigación ha logrado una importante contribución ya que se podría iniciar programas de remediación ambiental con bajos costos dado que se trata de una planta endémica; es decir totalmente adaptada para estas cuatro regiones. Dicho sea de paso, actualmente son consideradas, según el Ministerio de Energía y Minas, como las regiones que reciben más fondos por derecho de vigencia de minas.

De acuerdo con los resultados obtenidos en la tabla 5, se puede mencionar que la especie Croton Alnifolius, es una especie bio remediadoras. Pese a que no se ha ubicado una tabla que nos permita comprobar si estas plantas son hiper acumuladoras, de acuerdo con la teoría de dichas plantas y la observación de campo, se les podrían clasificar como especies hiper acumuladoras, ya que los híper acumuladores tienen la desventaja de ser pequeñas y tardar mucho en crecer. Situación que se observó en el lugar de investigación, ya que los individuos de Croton alnifolius que crecen sobre el relave son de dimensiones más pequeñas que aquellas que crecen en un ambiente no contaminado. 


\section{CONCLUSIONES}

Existen plantas que tienen capacidad de bioacumular cobre y zinc teniendo las siguientes cifras para la especie Croton alnifolius, la cual almacena 70.0 ppm para cobre y 1930.9 ppm para zinc con lo cual se afirmaría que la hipótesis ha sido comprobada positivamente con los datos obtenidos.

Se pudo identificar las plantas cuyos nombres científicos son: Crotón alnifolius, Atriplex rotundifolia, Alternanthera halimifolia, Bacharis Latifolia y Capparis Sp. Por el análisis de biomasa y repetición de individuos por especie se logró identificar que las 2 especies que mejor adaptación al medio demostraron fueron, en primer lugar, el Croton alnifolius que presentó más individuos en los 4 cuadrantes; seguido por la especie Atriplex rotundifolia de la cual se observaron 4 repeticiones.

De acuerdo con los resultados obtenidos en el estudio correspondiente al relave minero de la empresa Perú Bar en Corcona, se puede afirmar que dicho suelo tiene alto contenido de cobre y zinc y que las especies vegetales son plantas estacionarías, es decir, su abundancia está ligada a la humedad del hábitat en la que se halla, ya que en épocas en las de mayor presencia de humedad en el ambiente, mayor era el número de especies vivas. En cambio, cuando la humedad era menor, estas se encontraban escasas y marchitas.

Luego de los primeros análisis de laboratorio se determinó que el Croton alnifolius, además de adaptarse a su medio, podía también concentrar altas cantidades de cobre y zinc a diferencia de las otras especies. Los resultados de los análisis realizados al Croton alnifolius que crecía en suelo con relave en el resultado de la muestra control se demostró que las especies que crecen sobre el mismo acumulan alto contenido de zinc. Así, conforme a los resultados en las repeticiones del Croton alnifolius se puede definir como una especie con alto grado de bioacumulación y tolerancia al zinc, lo cual indicaría que esta especie podría servir como fitorremediadora en relaves que tengan similares condiciones.

Con la presencia de la especie Croton alnifolius en época seca se demostró su rusticidad, lo que demuestra que esta especie es resistente a las variables de temperatura y humedad.

\section{RECOMENDACIONES}

Para determinar cuál es el grado de recuperación que podrían tener los relaves contaminados con cobre y zinc, se recomienda continuar con esta investigación en un modo experimental controlando las variables, así se podría presentar esta especie con una fundamentación científica como fitorremediadora de suelos contaminados con zinc. 
Estudiar ambientes que se encuentren contaminados por relaves en los cuales se observe la presencia de especies vegetales para determinar su capacidad de Bioacumulacion de cobre y zinc.

Se recomienda realizar investigación durante la época húmeda para obtener datos y compararlos para observar el comportamiento de las especies bajo estos cambios en las variables.

Se recomienda desarrollar las características fenológicas de las plantas identificadas como bioacumuladoras, las cuales servirán para establecer las mejores técnicas de propagación para las plantas bioacumuladoras.

Se recomienda realizar este tipo de estudio en otras zonas de mayor altitud donde existen proyectos mineros.

\section{REFERENCIAS}

Bautista Zúñiga, Francisco. Introducción al estudio de la contaminación del suelo por metales pesados, 1 edición. México, UADY, 1999. 109pag.

Bayón, Fernández, Et, al. Evolución del asentamiento de especies utilizadas para la recuperación biológica de zonas alteradas por la minería. Arequipa - Perú, 2000. 274pag.

Castillo Francisco. Biotecnología ambiental. Madrid. editorial Tebar, 2005. 616pag.

Castillo Rodríguez, L. Uso de líquenes como bioindicadores en presencia de metales pesados en una zona de pasivos mineros en abandono. Universidad Agraria la Molina, 2011.

Diez Lázaro, Javier. Fitocorrección de suelos contaminados con metales pesados: evaluación de plantas tolerantes y optimización del proceso mediante prácticas agronómicas. Editor Universidad Santiago de Compostela, 1999.

Durán Cuevas Paola Andrea. Transferencia de metales de suelo a planta en áreas mineras: Ejemplos de los Andes peruanos y de la Cordillera Prelitoral Catalana. Barcelona, 2010.

El Peruano A: ley que regula los pasivos ambientales de actividad minera, julio, 2004. Página 271834.

El Peruano B: Decreto legislativo N 613-1990-minem, Setiembre, 1990.

El Peruano C: Decreto legislativo N757-1990, noviembre, 1991.

El Peruano D: Aprueban estándares de calidad ambiental para suelos. Decreto Supremo N002-2013-MINAM, marzo, 2013. Página 491497.

Eweis, Juana et, al. Principios de Biorrecuperación. Madrid, Mc Graw Hill, 1999, 327pag.

García, I., Dorronsoro, C. 2005. Contaminación por Metales Pesados. En Tecnología de Suelos. Universidad de Granada. Departamento de Edafología y Química Agrícola. En línea http://edafologia.ugr.es/conta/tema15/introd.htm.

Guzmán, Marta. La contaminación de suelos y aguas: su prevención con nuevas sustancias naturales. Madrid. Editorial universidad de Sevilla 2007.244pag. 
Kabata-Pendias, Alina. Elementos traza en suelo y plantas, tercera edición, Florida, EEUU, 2000. Editorial Francis y Taylor 432 pag.

Martin, C. Heavy Metals Trends in Floodplain Sediments and Valley Fill. Catena, 2000. pp 39, 53.

Moreno Jiménez, Eduardo. Recuperación de suelos mineros contaminados con arsénico mediante fitotecnologias. madrid,2010.

Rodríguez, Robert y GARCÍA Cortés, Ángel. Los Residuos Minero-Metalúrgicos en El Medio Ambiente. Vol. 11. Madrid, editorial IGME, 2006. 744pag.

SEMARNAT. Metales Pesados. México. En línea: http://www.ine.gob.mx.

Seoánez Calvo, Mariano. Contaminación de suelos: estudios, tratamiento y gestión. España, Ediciones mundi prensa. 1999.

Sociedad Nacional de Minería Petróleo y Energía (SNMPE). Impacto económico de la minería en el Perú. 2012, Perú. En línea: http://www.snmpe.org.pe

Talledo Rodríguez, G. evaluación de la capacidad de bioacumulación de metales pesados ( $\mathrm{Pb}, \mathrm{Cd}, \mathrm{Cu}$ y $\mathrm{Hg}$ ) en dos especies de hongos: agancus bisporus y Agaricus bunnences. Durante su periodo vegetativo. Universidad Agraria la Molina, 2008. 